\title{
Photodegradation and Aggregation Prevention of Natural Melanin Nanoparticles by Silica Coating Method
}

\author{
Yujia Liang, Lu Sun, Xungai Wang \\ Institute for Frontier Materials, Deakin University, Geelong, Australia \\ Email:1u.sun@deakin.edu.au
}

How to cite this paper: Liang, Y.J., Sun, L. and Wang, X.G. (2018) Photodegradation and Aggregation Prevention of Natural Melanin Nanoparticles by Silica Coating Method. Journal of Materials Science and Chemical Engineering, 6, 1-10. https://doi.org/10.4236/msce.2018.61001

Received: November 1, 2017

Accepted: January 1, 2018

Published: January 4, 2018

\begin{abstract}
Melanin, the main photoreceptor in living organics, is largely abundant in pigmented hair tissues. Its promising properties have been widely studied in order to fully explore the corresponding applications. However, the photodegradating and aggregating natures of melanin, to some extent, restrict the development of this eco-friendly biomaterial. This study is conducted to overcome both disadvantages by a mature method that coating the original melanin nanoparticles with silica as shells. The results revealed that the aggregation of the melanin/silica nanoparticles (MSNP) was decreased obviously by a $5 \mathrm{~h}$ coating and appeared a uniformly mono-dispersed solution. The MSNP that coated for $20 \mathrm{~h}$ provided an efficient protection on the photodegradation of the melanin with a $50 \%$ maintenance of the melanin content compared with $16 \%$ of original melanin and $2 \%$ of MSNP- $5 \mathrm{~h}$.
\end{abstract}

\section{Keywords}

Melanin Nanoparticles, Silica Coating, Photodegradation, Particle Aggregation

\section{Introduction}

Melanin is a general term of the ubiquitous biopolymeric pigment in living organisms [1]. Its intriguing characteristics and potential opportunities in many fields have drawn much attention from the scientists since a long time [1] [2] [3]. The advantages of melanin include photoprotector, free radical scavenger, metal ion stabilizer and electronic-ionic hybrid conductor [4] [5] [6] [7]. Correspondingly, melanin can be potentially applied to biomedicine, cosmetics, energy and surface modification [8] [9] [10]. Nevertheless, despite decades of in- 
vestigation, melanin is still regarded as a mysterious material due to the differential origins, complex synthetic pathways and inhomogeneous structures [11]. The unwished traits of melanin such as insolubility, photodegradation and aggregation also restrict the pace of fundamental research and practical applications [6] [12] [13].

Zareba et al. (2006) found that illumination could affect melanin from many aspects, such as destroy the morphologies of the melanin particles, reduce the pigment content to induce an irreversible photobleaching, and inhibit its antioxidant properties [14]. It is proved that solar irradiation with the existence of oxygen can lead to the oxidation of the hydroquinone-based units of melanin, resulting in a ring opening reaction and a reduction of its functional groups [15]. Melanin is thus photodegraded with deteriorated properties. The irradiation on melanin also causes the generation of harmful free radicals, one of them is the reactive oxygen species (ROS), which could lead to DNA damage in the organism or react with other substances [16]. Surprisingly, the aggregate behaviour of melanin, at this stage, plays a crucial role in gathering a large number of melanin radicals to scavenge those photoinduced ROS [17]. However, one concern is that the increase of melanin aggregation could lead to some age-related photochemistry changes [18]. Moreover, Kim et al. (2013) found that the aggregation can lower its optical absorbing ability which influenced the efficiency of the melanin-based energy storage device [8]. Therefore, proper melanin modifications are needed to overcome these limitations.

In this study, the Stöber method [19] was introduced to modify the naturally extracted melanin particles with silica, and the mono-dispersed core-shell melanin/silica structure was achieved. The silica coated melanin with different ageing duration was characterized and the effect of the coated melanin under irradiation was studied. Results showed that the melanin/silica nanoparticles (MSNP) have successfully prevented the particle aggregation, improved the particle dispersity in water and organic solutions, and enhanced the optical absorbing ability. The photodegradation of MSNP- $20 \mathrm{~h}$ solution has been dramatically slowed down with the aid of the completed silica shell and presented a uniformly descendant degradation rate.

To our knowledge, this is the first time when melanin is modified on the basis of keeping its original structure, and the prevention of its photodegradation, particle aggregation and deposition were realized at the same time. Hence, this work, to some extent, may open up a new perspective on the basic research of melanin and expand its potential applications.

\section{Materials and Experiments}

\subsection{Materials}

Brown alpaca fibres were collected from Australia pasture and were thoroughly cleaned by acetone/ethanol mixture prior to use. Hydrochloric acid ( $\mathrm{HCl}, 32 \%)$, tetraethyl orthosilicate (TEOS, 98\%), ammonium hydroxide (30\%), ethanol 
(100\%), acetone (100\%), methyl methacrylate (MMA, 99\%), and 1,1'-Azobis (cyclohexanecarbonitrile) (AICN, 98\%) were purchased from Sigma-Aldrich.

\subsection{Melanin Extraction}

The extraction method was conducted using the reported acid hydrolysis method [2]. Typically, $2 \mathrm{~g}$ of the brown fibres were mixed with $20 \mathrm{~mL}$ of $\mathrm{HCl}$ (1\%) in a round-bottom flask and stirred for $5 \mathrm{~min}$ to remove the water-soluble components. After centrifugation, the precipitates were immersed in $80 \mathrm{~mL}$ of $32 \% \mathrm{HCl}$ and heated at $100^{\circ} \mathrm{C}$ in an oil bath with mechanical stirring for $3 \mathrm{~h}$. The precipitates were then washed with deionized water and centrifuged at 4000 rpm for 5 min repeatedly until the neutral $\mathrm{pH}$ of the suspension was approached. The supernatant was removed and the samples were vacuum dried at room temperature in the dark.

\subsection{Preparation of Melanin/Silica Nanoparticles (MSNP)}

The silica coating (SC) method was employed as described in the previous work [20]. Specifically, $10 \mathrm{mg}$ of the melanin was dispersed uniformly in $1.5 \mathrm{~mL}$ of distilled water and $1.5 \mathrm{~mL}$ of ethanol. $0.4 \mathrm{~mL}$ of ammonia was mixed in $6 \mathrm{~mL}$ ethanol and poured into the melanin solution. After mechanical stirring for 10 min, a mixture of $0.05 \mathrm{~mL}$ of TEOS and $2 \mathrm{~mL}$ of ethanol was added dropwise. The final products were obtained after different stirring duration ( $5 \mathrm{~h}$ and $20 \mathrm{~h}$ ) and were then washed with deionized water for three times, followed by drying at room temperature in the dark.

\subsection{Characterizations}

The core-shell structures of MSCP before and after SC were examined on a JEOL 2100 LaB6 TEM. The size and distribution of MSNP were measured by Dynamic light scattering (DLS) on Malvern Zetasizer. Samples were dispersed in deionized water, placed in low volume disposable cuvettes and measured under size mode. The absorption properties of the melanin solutions before and after SC were scanned with a Cary 3 UV-Vis spectrometer from 200 to $800 \mathrm{~nm}$. The Fourier transform infrared spectroscopy (FTIR) spectra were recorded on a Bruker FTIR Spectrophotometer with ATR accessories from 4000 to $600 \mathrm{~cm}^{-1}$ under the accumulation of 32 scans at $4 \mathrm{~cm}^{-1}$ resolution. The simulated solar irradiation test was conducted with an Atlas Suntester XLS instrument that equipped with a $1500 \mathrm{~W}$ xenon arc lamp (light range: $300-800 \mathrm{~nm}$ ). The irradiation dose was $350 \mathrm{~W} / \mathrm{m}^{2}$ and the chamber temperature was $35^{\circ} \mathrm{C}$. The solutions were under mechanical stirring during irradiation and the concentrations were kept same. $1 \mathrm{~mL}$ of each solution was taken out every $10 \mathrm{~h}$ and scanned with UV-Vis spectroscopy.

\section{Results and Discussion}

\subsection{Characterizations of the MSNP}

The morphologies of the original melanin and MSNP are shown in Figure 1. 

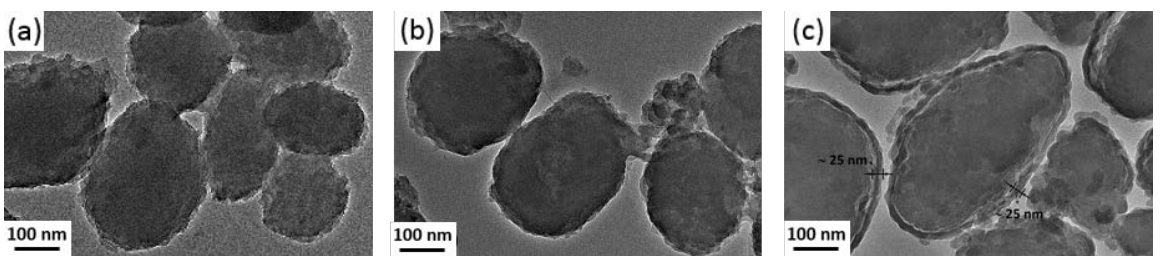

Figure 1. TEM images of the (a) original melanin, (b) MSNP-5 $\mathrm{h}$ and (c) MSNP-20 h.

The original melanin particles exhibit round or elliptical shapes with distinct sizes, which is inconsistent with the extracted melanin from other mammal's hair samples in previous research [21] [22]. It can be seen that the particle surface is cratered instead of smooth which is due to the special bio-synthetic pathway and inhomogeneous nature of melanin that polymerizing the oligomers with a variety of units through different of bonds, such as $\pi$-stacking interactions [23]. Figure 1(b) of the MSNP- $5 \mathrm{~h}$ barely changes compare with the original melanin in addition to the appearance of the exotic substances, meaning the formation of the silica shell outside melanin particles. The particles also seem to be separated apart from each other at this stage. When the coating duration reached $20 \mathrm{~h}$, a conspicuous melanin core and silica shell structure was obtained (as shown in Figure 1(c)). The formation of the silica shell was slowly, continuously and evenly owing to the well-studied Stöber method. Specifically, the addition of ammonia assists the synthesis of silica from TEOS, and the $\mathrm{NH}_{4}{ }^{+}$that adsorbs onto melanin helps to get a positively charged surface so that the hydrogen bond could be formed between $\mathrm{NH}_{4}^{+}$and the $\mathrm{OH}^{-}$of silica. Then, the continuous hydrolysis and condensation reactions took place in this alkali system, thereby achieving a uniform silica layer outside the melanin particles [24].

DLS measurement exhibits the distribution of the three particles in aqueous solutions (Figure 2). Apparently, the DLS result shows that original melanin is very easy to get serious particle aggregation, as described in previous study [25]. However, a $5 \mathrm{~h}$ coating can largely decrease the aggregation of melanin, showing a particle size distribution from 200 to $1000 \mathrm{~nm}$ and an average size of $480 \mathrm{~nm}$ which is consistent with the TEM result (Figure $1(\mathrm{~b})$ ). The average particle size of MSNP-20 h becomes $530 \mathrm{~nm}$ with a $20 \mathrm{~h}$ coating. The increase contributes to the growing of the silica shell thickness and may imply the average shell thickness is around $25 \mathrm{~nm}$ (Figure 1(c)). The photo of solutions of MSNP-5 h and MSNP-20 h after standing overnight demonstrate again the welldispersity of melanin solution (inset of Figure 2).

The typical UV-Vis spectra of melanin can be obtained from the MSNP solution (Figure 3(a)) [26] and the UV-Vis spectrum of MSNP-20 h shows an increasing UV absorbing ability compared with the original melanin and MSNP-5 $\mathrm{h}$ at the same concentration, which probably owing to the reduced particle aggregating phenomenon that enhanced the overall particle surface area and solution dispersity [27].

The representative functional groups in the FTIR spectrum of the original melanin coincides well with the reported work (Figure 3(b)) [28] [29], including 


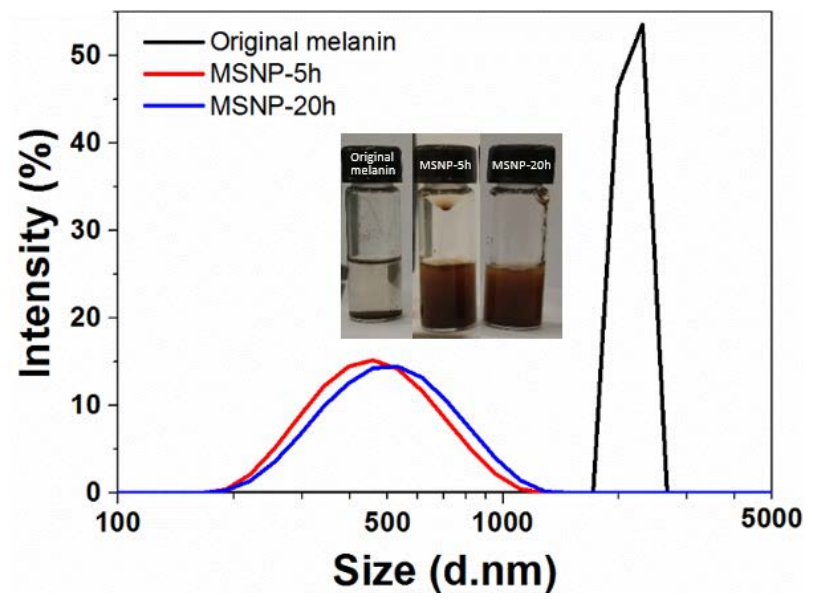

Figure 2. DLS spectra of the original and MSNP solutions. Insets: the optical photos of the solutions stand overnight in the dark.

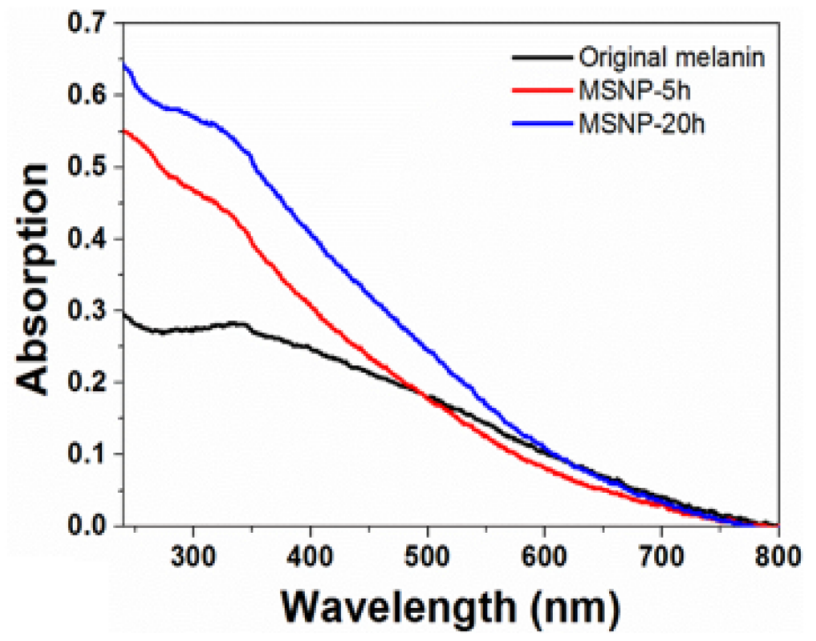

(a)

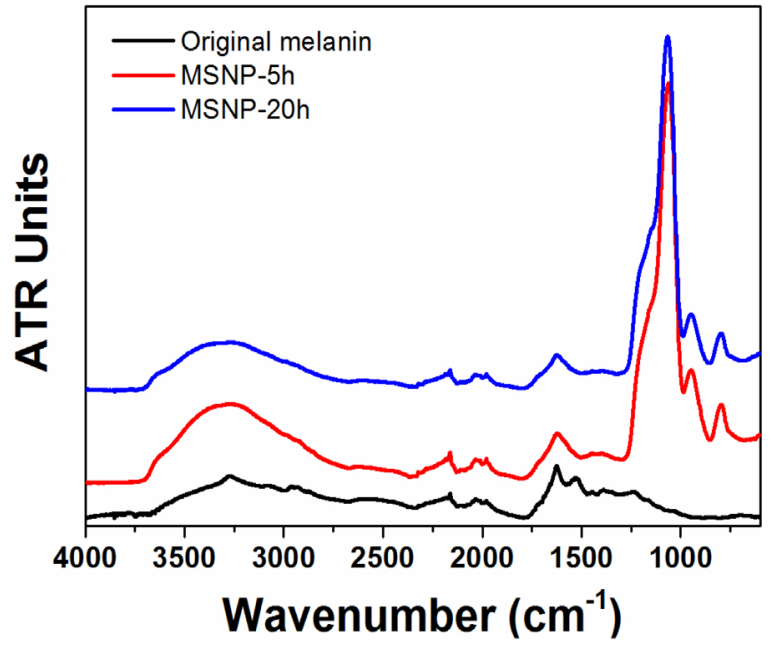

(b)

Figure 3. (a) UV-Vis and (b) FTIR spectra of the original and MSNP solutions. 
the $-\mathrm{OH},-\mathrm{NH}_{2}$ groups at around $3400 \mathrm{~cm}^{-1}$, and aromatic $\mathrm{C}=\mathrm{C}, \mathrm{COO}-, \mathrm{C}=\mathrm{O}$ groups at $1600 \mathrm{~cm}^{-1}$. The spectra of the MSNP samples exhibit significant difference below $1300 \mathrm{~cm}^{-1}$. Especially, a strong peak at around $1070 \mathrm{~cm}^{-1}$ refers linearsilica structure (Si-O-Si), a weak peak at $796 \mathrm{~cm}^{-1}$ represents the network of silica [30], and a gentle slope at around $1220 \mathrm{~cm}^{-1}$ indicates the unreacted TEOS residues [31].

\subsection{Photodegradation of the MSNP}

It has been proved that coated with silica shell could inhibit the photocatalytic activity of the core material from irradiation to some extent [20] [32]. Therefore, the photoprotective effect of the SC on melanin particles is studied. The degradation trends of each melanin sample with increasing dose of simulated solar irradiation are shown in Figure 4. The $\mathrm{A} / \mathrm{A}_{0}$ refers to the ratio of the absorption after specific irradiation duration to the initial absorption at the wavelength of $500 \mathrm{~nm}$. It can be seen that all of the three samples showed an overall downtrend after irradiation.

Specifically, the original melanin has barely degraded for the first $10 \mathrm{~h}$ irradiation. This probably caused by the severe aggregation of the melanin particles in the solution that slowed down the photo-induced impact on thesingle particle. On the contrary, a serious photodegradation (30\%) of the MSNP- $5 \mathrm{~h}$ took place at the first $10 \mathrm{~h}$. In terms of the DLS tests, MSNP- $5 \mathrm{~h}$ has already resulted in a great improvement of particle aggregation compared with the original melanin, but the TEM images and UV-Vis spectra indicated that the silica shell could not be fully formed for a $5 \mathrm{~h}$ coating. Therefore, the overall degradation trend of the aggregated original melanin is slower than the well separated but uncompleted MSNP-5 h. Notably, the MSNP-20 h showed a linearly degrading rate, and is much slower than the other solutions after irradiation for $30 \mathrm{~h}$. This may owe to the uniformly dispersed MSNP that could absorb the light evenly, causing the

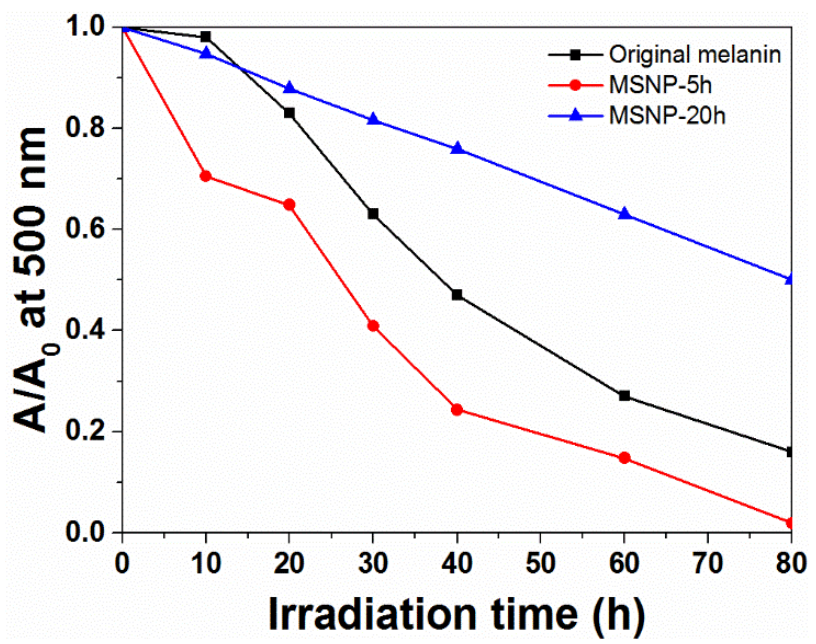

Figure 4. The photodegradation profile of original melanin, MSNP-5 $\mathrm{h}$ and MSNP-20 $\mathrm{h}$ solutions at $500 \mathrm{~nm}$. 

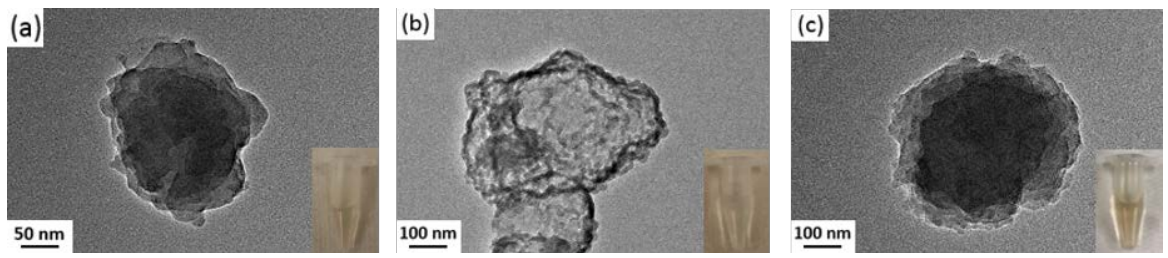

Figure 5. The TEM images of the (a) original melanin, (b) MSNP-5 $\mathrm{h}$ and (c) MSNP-20 $\mathrm{h}$ that irradiated for $80 \mathrm{~h}$. Insets: the optical photos of the corresponding solutions.

constant degradation speed. After exposure for $80 \mathrm{~h}$, the melanin in MSNP-5 $\mathrm{h}$ has almost been exhausted, while less than $20 \%$ of the original melanin remained. Meanwhile, around 50\% of the melanin within MSNP-20h has been protected, suggesting the efficiency of using the silica coating method on suppressing melanin from photodegradation.

Figure 5 displays the TEM images and optical photos of the $80 \mathrm{~h}$ irradiated samples. It is found that the original melanin particle has been largely shrunken (Figure 5(a)), and the boundary of the particle surface is getting vague and out of shape, which can reflect the irreversible degradation effect of the irradiation on melanin. Similarly, the MSNP-5 h in Figure 5(b) presents a small dark core inside the silica shell. The shape of the shell still maintained as an original melanin particle but become transparent due to the shrinkage of the melanin core. By comparison, the MSNP-20 h (Figure 5(c)) shows more melanin contained within the shell, indicating a better protection provided by this structure.

The colors of the irradiated solutions, on the other side, intuitionally supported the conclusions, which are darkened from MSNP-5 h (white), original melanin (pale yellow) to MSNP-20 h (yellow). The fading color coincident well with the UV-Vis results.

\section{Conclusion}

In this study, silica coating (SC) method has been successfully applied to natural melanin nanoparticles that extracted from pigmented alpaca fibers. The silica shell is formed outside of the melanin particle surface slowly and uniformly, leading to an improved particle aggregation and solution dispersity. The mela$\mathrm{nin} /$ silica nanoparticles (MSNP) that coated for $20 \mathrm{~h}$ efficiently inhibited the photodegradation phenomenon. $50 \%$ of the melanin could be maintained after an $80 \mathrm{~h}$ irradiation when the melanin content in other samples only remained less than $20 \%$.

In light of this fundamental research on the modification of melanin nanoparticles and the achievement of mono-dispersed melanin solution, more possibilities could be addressed by applying this environmentally benign biomaterial to multiple areas and realize its practical applications.

\section{Acknowledgements}

The $1^{\text {st }}$ author would like to acknowledge the Ph.D. scholarship provided by 
Deakin University.

\section{References}

[1] d'Ischia, M., Wakamatsu, K., Cicoira, F., Di Mauro, E., Garcia-Borron, J.C., Commo, S., Galván, I., Ghanem, G., Kenzo, K. and Meredith, P. (2015) Melanins and Melanogenesis: from Pigment Cells to Human Health and Technological Applications. Pigment Cell \& Melanoma Research, 28, 520-544. https://doi.org/10.1111/pcmr.12393

[2] Song, R., Li, R., Yu, Q. and Deng, S. (2011) Effects of Different Refining Methods on the Micro-Morphology, Spectra Properties and Metal Elements of Melanin from Squid Ink. Oceanologia ET Limnologia Sinica, 42, 839-843.

[3] Liu, Y., Ai, K. and Lu, L. (2014) Polydopamine and Its Derivative Materials: Synthesis and Promising Applications in Energy, Environmental, and Biomedical Fields. Chemical Reviews, 114, 5057-5115. https://doi.org/10.1021/cr400407a

[4] Mostert, A.B., Powell, B.J., Pratt, F.L., Hanson, G.R., Sarna, T., Gentle, I.R. and Meredith, P. (2012) Role of Semiconductivity and Ion Transport in the Electrical Conduction of Melanin. Proceedings of the National Academy of Sciences, 109, 8943-8947. https://doi.org/10.1073/pnas.1119948109

[5] Brenner, M. and Hearing, V.J. (2008) The Protective Role of Melanin against UV Damage in Human Skin. Photochemistry and Photobiology, 84, 539-549.

https://doi.org/10.1111/j.1751-1097.2007.00226.x

[6] Ju, K.-Y., Lee, Y., Lee, S., Park, S.B. and Lee, J.-K. (2011) Bioinspired Polymerization of Dopamine to Generate Melanin-Like Nanoparticles Having an Excellent Free-Radical-Scavenging Property. Biomacromolecules, 12, 625-632. https://doi.org/10.1021/bm101281b

[7] Liu, Y. and Simon, J.D. (2005) Metal-Ion Interactions and the Structural Organization of Sepia Eumelanin. Pigment Cell Research, 18, 42-48. https://doi.org/10.1111/j.1600-0749.2004.00197.x

[8] Kim, Y.J., Wu, W., Chun, S.E., Whitacre, J.F. and Bettinger, C.J. (2013) Biologically Derived Melanin Electrodes in Aqueous Sodium-ion Energy Storage Devices. Proceedings of the National Academy of Sciences, 110, 20912-20917. https://doi.org/10.1073/pnas.1314345110

[9] Manivasagan, P., Venkatesan, J., Sivakumar, K. and Kim, S.K. (2013) Actinobacterial Melanins: Current Status and Perspective for the Future. World Journal of Microbiology \& Biotechnology, 29, 1737-1750. https://doi.org/10.1007/s11274-013-1352-y

[10] Nam, H.J., Kim, B., Ko, M.J., Jin, M., Kim, J.M. and Jung, D.Y. (2012) A New Mussel-Inspired Polydopamine Sensitizer for Dye-Sensitized Solar Cells: Controlled Synthesis and Charge Transfer. Chemistry-A European Journal, 18, 14000-14007. https://doi.org/10.1002/chem.201202283

[11] Sarna, T. and Swartz, H.A. (2006) The Physical Properties of Melanins. The Pigmentary System: Physiology and Pathophysiology, Second Edition, 311-341. https://doi.org/10.1002/9780470987100.ch16

[12] Ju, K.Y., Lee, Y., Lee, S., Park, S.B. and Lee, J.K. (2011) Bioinspired Polymerization of Dopamine to Generate Melanin-Like Nanoparticles Having an Excellent FreeRadical-Scavenging Property. Biomacromolecules, 12, 625-632. https://doi.org/10.1021/bm101281b

[13] Sarna, T., Burke, J.M., Korytowski, W., Różanowska, M., Skumatz, C.M., Zaręba, A. and Zaręba, M. (2003) Loss of Melanin from Human RPE with Aging: Possible Role 
of Melanin Photooxidation. Experimental Eye Research, 76, 89-98. https://doi.org/10.1016/S0014-4835(02)00247-6

[14] Zareba, M., Szewczyk, G., Sarna, T., Hong, L., Simon, J.D., Henry, M.M. and Burke, J.M. (2006) Effects of Photodegradation on the Physical and Antioxidant Properties of Melanosomes Isolated from Retinal Pigment Epithelium. Photochemistry and Photobiology, 82, 1024-1029. https://doi.org/10.1562/2006-03-08-RA-836

[15] Korytowski, W. and Sarna, T. (1990) Bleaching of Melanin Pigments. Role of Copper Ions and Hydrogen Peroxide in Autooxidation and Photooxidation of Synthetic dopa-Melanin. The Journal of Biological Chemistry, 265, 12410-12416.

[16] Dunford, R., Salinaro, A., Cai, L., Serpone, N., Horikoshi, S., Hidaka, H. and Knowland, J. (1997) Chemical Oxidation and DNA Damage Catalysed by Inorganic Sunscreen Ingredients. FEBS Letters, 418, 87-90. https://doi.org/10.1016/S0014-5793(97)01356-2

[17] Seagle, B.-L.L., Rezai, K.A., Kobori, Y., Gasyna, E.M., Rezaei, K.A. and Norris, J.R. (2005) Melanin Photoprotection in the Human Retinal Pigment Epithelium and Its Correlation with Light-Induced Cell Apoptosis. Proceedings of the National Academy of Sciences of the United States of America, 102, 8978-8983. https://doi.org/10.1073/pnas.0501971102

[18] Boulton, M., Różanowska, M. and Różanowski, B. (2001) Retinal Photodamage. Journal of Photochemistry and Photobiology B: Biology, 64, 144-161. https://doi.org/10.1016/S1011-1344(01)00227-5

[19] Stöber, W., Fink, A. and Bohn, E. (1968) Controlled Growth of Monodisperse Silica Spheres in the Micron Size Range. Journal of Colloid and Interface Science, 26, 62-69. https://doi.org/10.1016/0021-9797(68)90272-5

[20] Zhang, M., Xie, W., Tang, B., Sun, L. and Wang, X. (2016) Synthesis of TiO2\&SiO2 Nanoparticles as Efficient UV Absorbers and Their Application on Wool. Textile Research Journal, 0040517516659375.

[21] Menon, I. A., Persad, S., Haberman, H.F. and Kurian, C.J. (1983) A Comparative Study of the Physical and Chemical Properties of Melanins Isolated from Human Black and Red Hair. Journal of Investigative Dermatology, 80, 202-206. https://doi.org/10.1111/1523-1747.ep12534045

[22] Fan, R., Yang, G. and Dong, C. (2010) Study of Hair Melanin in Various Hair Color Alpaca (Lama pacos). Asian-Australasian Journal of Animal Sciences, 23, 444-449. https://doi.org/10.5713/ajas.2010.90333

[23] Clancy, C.M. and Simon, J.D. (2001) Ultrastructural Organization of Eumelanin from Sepia Officinalis Measured by Atomic Force Microscopy. Biochemistry, 40, 13353-13360. https://doi.org/10.1021/bi010786t

[24] Gui-Long, X., Changyun, D., Yun, L., Pi-Hui, P., Jian, H. and Zhuoru, Y. (2011) Preparation and Characterization of Raspberry-like SiO2 Particles by the Sol-Gel Method. Nanomaterials and Nanotechnology, 1, 21. https://doi.org/10.5772/45813

[25] Eisner, M. (1992) The Physical and Chemical Properties of Eye Melanin and Their Relationship to Photoprotection. Occhio e Radiazoni Solari, Tecnologie Fotoprotettive e Funzione Visiva. M. Cordella, editor. Universita degli Studi di Parma, Parma, Italy, 61-73.

[26] Riley, P.A. (1997) Melanin. The International Journal of Biochemistry and Cell Biology, 29, 1235. https://doi.org/10.1016/S1357-2725(97)00013-7

[27] Xian-Fu, W., Na, W., Xiao-Fang, W., Yan-Ni, Y. and Bei-Qing, H. (2009) Evaluation of the Pigment Dispersion of UV-Curable Inkjet Ink Using UV Absorption Spec- 
trum. In: NIP \& Digital Fabrication Conference, Vol. 2009, Society for Imaging Science and Technology, 323-326.

[28] Yao, Z., Qi, J. and Wang, L. (2012) Isolation, Fractionation and Characterization of Melanin-Like Pigments from Chestnut (Castanea mollissima) Shells. Journal of Food science, 77, 671-676. https://doi.org/10.1111/j.1750-3841.2012.02714.x

[29] Liu, Y., Hong, L., Wakamatsu, K., Ito, S., Adhyaru, B., Cheng, C.Y., Bowers, C.R. and Simon, J.D. (2005) Comparison of Structural and Chemical Properties of Black and Red Human Hair Melanosomes. Photochemistry and Photobiology, 81, 135144. https://doi.org/10.1562/2004-08-03-RA-259.1

[30] Serizawa, T., Hamada, K.-i., Kitayama, T., Fujimoto, N., Hatada, K. and Akashi, M. (2000) Stepwise Stereocomplex Assembly of Stereoregular Poly (Methyl Methacrylate) s on a Substrate. Journal of the American Chemical Society, 122, 1891-1899.

[31] Chan, C., Peng, S., Chu, I. and Ni, S. (2001) Effects of Heat Treatment on the Properties of Poly(Methyl Methacrylate)/Silica Hybrid Materials Prepared by Sol-Gel Process. Polymer, 42, 4189-4196. https://doi.org/10.1016/S0032-3861(00)00817-X

[32] Zhang, M., Tang, B., Sun, L. and Wang, X. (2016) Protection of Silica-Coated ZnO Nanoparticles on Pre-Dyed Polyester Fabrics Against Photofading. The Journal of the Textile Institute, 1-8. 